\title{
Radiation-Driven Warping: The Origin of Warps and Precession in Accretion Disks
}

\author{
Philip R. Maloney ${ }^{1}$, Mitchell C. Begelman ${ }^{2,3}$ and J. E. Pringle ${ }^{4}$
}

\begin{abstract}
A geometrically thin, optically thick, warped accretion disk with a central source of luminosity is subject to non-axisymmetric forces due to radiation pressure; the resulting torque acts to modify the warp. In a recent paper, Pringle (1996) used a local analysis to show that initially planar accretion disks are unstable to warping driven by radiation torque. Here we extend this work with a global analysis of the stable and unstable modes. We confirm Pringle's conclusion that thin centrally-illuminated accretion disks are generically unstable to warping via this mechanism; we discuss the time-evolution and likely steady-state of such systems and show specifically that this mechanism can explain the warping of the disk of water masers in NGC 4258 and the 164-day precession period of the accretion disk in SS 433. Radiation-driven warping and precession provides a robust mechanism for producing warped, precessing accretion disks in active galactic nuclei and X-ray binary systems.
\end{abstract}

Subject headings: accretion disks - instabilities - galaxies: individual (NGC 4258) stars: individual (SS 433)

\section{Introduction}

An optically thick accretion disk which is illuminated by the central source of luminosity will reradiate the intercepted flux essentially normal to the local disk surface. As first noted by Petterson (1977), if the accretion disk is warped (i.e., has a non-constant inclination and/or

\footnotetext{
${ }^{1}$ Center for Astrophysics and Space Astronomy, University of Colorado, Boulder, CO 80309-0389; maloney@shapley.colorado.edu

${ }^{2}$ JILA, University of Colorado and National Institute of Standards and Technology, Boulder, CO 80309-0440; mitch@jila.colorado.edu

${ }^{3}$ Also at Department of Astrophysical, Planetary and Atmospheric Sciences, University of Colorado

${ }^{4}$ Institute of Astronomy, Madingley Road, Cambridge CB3 0HA, England
} 
orientation of the line of nodes as a function of radius), then the resulting radiation pressure force will be non-axisymmetric, leading to torquing of the disk and modification of the warp.

The origin of this radiation torque can be seen as follows. Consider an annulus of an accretion disk with a central source of radiation. If we warp this annulus slightly, it will be illuminated by the central source, and, if the disk is optically thick, it will absorb the incident flux. The momentum associated with this absorbed incident flux is purely radial, and so the force due to the incident radiation will produce zero torque. If the disk is optically thick to re-emission of the absorbed radiation, however, the net re-emitted flux will be normal to the local plane of the disk. The radiation pressure acting on the irradiated surface due to the re-emitted radiation will therefore exert a torque at each exposed point of the annulus. If the annulus is warped, i.e., the local angle of tilt varies with azimuth, then the net torque on the ring will be non-zero, because the deposition of radiation momentum per unit area of the ring will be non-axisymmetric. In general, the net torque will be non-zero provided that there is a radial gradient in either the tilt angle $\beta$ (in which case the ring will precess) or the angle of the line of nodes (in which case the torque will cause the tilt to change). Note that the disk must be optically thick both to absorption of the incident radiation and to re-radiation of the absorbed flux. If the disk is optically thin to absorption, then the absorption rate will be essentially constant through the annulus, and the flux re-radiated from the two faces of the annulus (the irradiated face and the opposite face, which does not see the central source directly) will be the same, so that the re-emitted radiation will exert zero torque. If the disk is optically thin to re-emission, then the re-emitted radiation will be isotropic, and again the torque will be zero.

Petterson (1977) calculated the influence of this radiation torquing on the disk shape, assuming an initially warped disk. However, Pringle (1996) has shown that even initially planar disks are unstable to warping due to radiation pressure, so that warping should be generic in centrally-illuminated accretion disks.

Pringle (1996) (hereafter P96) studied the disk dynamics using a local (WKB-type) analysis, in which the variation of the radiation torque parameter and viscosity with radius $R$ was ignored. However, such an approach is only marginally justified, since the unstable modes in Pringle's analysis have radial wavenumber $k \sim 2 \pi R^{-1}$. In this paper we extend the work of P96 in a global analysis; we show that this is in fact analytically soluble, with both stable "normal" modes and growing modes. In section 2 we solve the linearized equation for the evolution of the disk tilt, while in section 3 we compare our results to P96 and discuss the time evolution and likely steady state of such a system. We show in section 1 that this instability provides a plausible explanation for the warp observed in the Keplerian disk of water masers in the nucleus of NGC 4258, and for the 164-day precession period in SS 433. 


\section{Analysis of the Disk Dynamics}

Following P96, we define $\beta$ to be the local angle of tilt of the disk with respect to the normal to the equatorial plane, while $\gamma$ describes the orientation of the line of nodes with respect to a fixed axis in the equatorial plane. The equation for the evolution of the local tilt vector, $\mathbf{l}(R, t)=(\sin \beta \cos \gamma, \sin \beta \sin \gamma, \cos \beta)$ was derived by Pringle (1992) (his equation 2.5). We assume an isothermal, gas pressure-supported disk at $R \gg R_{s}\left(R_{s}\right.$ is the Schwarzschild radius), for which the surface density $\Sigma \propto R^{-3 / 2}$ and the radial velocity $V_{R}=\nu_{1} \Omega^{\prime} / \Omega$, where primes denote derivatives with respect to $R ; \nu_{1}$ is the usual disk viscosity, while we denote the viscosity which acts to reduce the disk tilt by $\nu_{2}$. This simplifies the evolution equation considerably ( $c f$. P96), and, assuming that $\beta \ll 1$ and $\beta^{\prime} R \ll 1$, it can be written compactly as an equation for $W \equiv \beta e^{i \gamma}$,

$$
\frac{\partial W}{\partial t}=-i \Gamma \frac{\partial W}{\partial R}+\frac{\partial}{\partial R}\left(\frac{1}{2} \nu_{2} \frac{\partial W}{\partial R}\right)
$$

where the radiation torque term is

$$
\Gamma=\frac{L}{12 \pi \Sigma R^{2} \Omega c}
$$

with $L$ the luminosity of the central source. We make the assumption that the disk is isothermal, i.e., it has the same temperature at all radii. Then, if the usual Shakura-Sunyaev viscosity parameter $\alpha$ is constant, we find that the viscosity $\nu_{1}$ scales as $\nu_{1}=\nu_{o}\left(R / R_{o}\right)^{3 / 2}$ where $R_{o}$ is an

arbitrary fiducial radius. We further assume that the ratio $\eta=\nu_{2} / \nu_{1}$ is constant. Since the surface density $\Sigma=\dot{M} / 3 \pi \nu_{1}$, we can express $\Gamma$ as

$$
\Gamma=\frac{\epsilon}{2 \sqrt{2} R_{s}^{1 / 2}} \frac{\nu_{o}}{R_{o}^{3 / 2}} R \equiv \Gamma_{o} R
$$

where we have assumed the luminosity is powered by accretion; the radiative efficiency $\epsilon \equiv L / \dot{M} c^{2}$. Equation (1) can then be written as

$$
\frac{\partial W}{\partial t}=-i \Gamma_{o} R \frac{\partial W}{\partial R}+\frac{1}{2} \frac{\eta \nu_{o}}{R_{o}^{3 / 2}} \frac{\partial}{\partial R}\left(R^{3 / 2} \frac{\partial W}{\partial R}\right) .
$$

\subsection{Particular Solutions}

For illustration we consider two particular solutions of equation (4).

a) The steady-state solution, in which the disk has constant structure in an inertial frame, can be obtained by setting $\partial W / \partial t=0$. The general solution for $W(R)$ is then given by

$$
W(R)=c_{1}+c_{2} \int_{0}^{R} R^{-3 / 2} \exp \left(\frac{4 i \Gamma_{o} R_{o}^{3 / 2}}{\eta \nu_{o}} R^{1 / 2}\right) d R,
$$

where $c_{1}$ and $c_{2}$ are arbitrary (complex) constants. Defining

$$
z=\frac{4 i \Gamma_{o} R_{o}^{3 / 2}}{\eta \nu_{o}} R^{1 / 2}=i \frac{\sqrt{2} \epsilon}{\eta}\left(R / R_{s}\right)^{1 / 2}
$$


(note that $z$ is pure imaginary) this can be written as

$$
W(z)=c_{1}+c_{2}^{\prime}\left[\frac{e^{i z}}{z}+i \int_{z}^{\infty} \frac{e^{i s}}{s} d s\right]
$$

where $c_{2}^{\prime}$ is arbitrary. Thus the steady disk has two independent solutions. The first, $W=$ constant, corresponds to the trivial solution of a flat disk misaligned with the axial plane. The second solution is (formally) singular at the origin $\left(W \sim R^{-1 / 2}\right.$ as $\left.R \rightarrow 0\right)$. More importantly, we require that the viscous torque exerted by each annulus of the disk on its neighbor, given by $G \propto R^{3 / 2} \partial W / \partial R$, is finite as $r \rightarrow 0$. The second solution therefore requires a source of angular momentum at the origin.

b) The 'constant-torque' solution is given by

$$
W(R, t)=e^{i \Gamma_{o} t / 2} R^{-1 / 2} .
$$

This is the solution that results when the viscous torque $G$ is independent of radius. In this case the disk as a whole precesses with angular velocity $\Gamma_{o} / 2$. The nodes all lie in the same radial direction, and the disk is stationary in a frame which rotates with angular velocity $\Gamma_{o} / 2$. This comes about because the radial dependence of $W$ insures that the precession rate induced at each radius is independent of radius ( $c f$. equation [3.10] in $\mathrm{P} 96)$.

This is actually a special case of a more general class of solutions, $W(R, t)=e^{i \Gamma_{o} t / a} R^{-1 / a}$, where $a \neq 0$. The difference is that for these solutions $\eta=\nu_{2} / \nu_{1}$ is not constant, as $\nu_{2} \propto R^{1+1 / a}$.

\subsection{More General Solutions}

In order to investigate more general time dependence, we Fourier transform with respect to time and write $\partial W / \partial t=i \sigma W ; \sigma$ in general has both real and imaginary parts. We again make the substitution (6) to rewrite equation (4) in the form

$$
z \frac{\partial^{2} W}{\partial z^{2}}+(2-z) \frac{\partial W}{\partial z}-\frac{2 \sigma}{\Gamma_{o}} W=0 .
$$

This is Kummer's equation (e.g., Abramowitz \& Stegun 1964, (AS), equation 13.1.1), $z W^{\prime \prime}+(b-z) W^{\prime}-a W=0$, with $b=2$ and $a=2 \sigma / \Gamma_{o}$. The solutions to Kummer's equation are confluent hypergeometric functions, $M(a, b, z)$ and $U(a, b, z)$. The condition that zero torque is

applied to the disk at the origin now becomes $z^{2} W^{\prime} \rightarrow 0$ as $z \rightarrow 0$. However, as $|z| \rightarrow 0, U \sim|z|^{1-b}$ and thus the contribution of the Kummer $U$ function provides a finite torque at the origin. For physical reasons we exclude the $U$ function from the solution. Thus the spatial part of $W$ is just given by the Kummer $M$ functions. Using the asymptotic expression for $M(a, b, z)$ (AS 13.5.1), we find that imposing the condition $W(z) \rightarrow 0$ as $z \rightarrow \infty$ requires that the real part of $a$ satisfy

$$
0<\operatorname{Re}(a)<2, \quad \text { that is, } \quad 0<\operatorname{Re}(\sigma)<\Gamma_{o} .
$$


This constraint on $\operatorname{Re}(a)$ is also required if the intercepted flux $d L$ (see equation (2.13) of Pringle (1996) ) is to remain finite as $z \rightarrow \infty$. It is not possible to impose the stronger regularity condition that $\left|z^{2} W\right| \propto|R W|=|\beta R|$ (i.e., the height of the warp above the untilted equatorial plane) remain finite as $z \rightarrow \infty$, as $z^{2} W$ diverges for all $\operatorname{Re}(a)$. Real astrophysical disks will always have finite $R W$, however, either due to physical truncation of the disk or because the surface density declines to the point where the disk becomes optically thin to the bulk of the incident flux.

A particular case of interest occurs when $\operatorname{Re}(a)=1$, that is, $a=1-i \omega$, with $\omega$ real. This corresponds to a steady precession of the disk structure with angular velocity $\Gamma_{0} / 2$, and an exponential growth of disk structure at a rate $e^{\omega t}$. In this case the $M$ functions are simply related to the $L=0$ Coulomb functions:

$$
M(1-i \omega, 2,2 i x)=e^{i x} F_{0}(\omega, x) x^{-1} / C_{0}(\omega)
$$

(AS 13.6.8) where $\omega$ can be either positive or negative and $F_{0}$ is the regular Coulomb wave function with $L=0$ (AS 14.1.4; note that both $\omega$ and $x$ are real). The azimuth of the line of nodes is given by $\gamma=x$, i.e., $\gamma \propto R^{1 / 2}$, which corresponds to a prograde spiral (cf. P96).

If $\omega=0$, there is no growth and the disk structure is steady in the precessing frame. In this case $M$ simplifies to the solution

$$
M(1,2,2 i x)=e^{i x} \frac{\sin (x)}{x}
$$

It is interesting to compare this solution (zero torque at the origin) with the torqued solution, equation (8), found above. Both solutions precess at the same rate, $\Gamma_{o} / 2$, and both solutions display an envelope to $|W|$ of the functional form $R^{-1 / 2}$. In both cases, the precession is driven solely by radiation (and thus the precession rate depends only on $\Gamma_{o}$ ). However, the zero torque solution maintains its constant shape (in the precessing frame) by using viscous forces, which would tend to smooth out the oscillations of $\beta$ with radius, to balance the radiation induced growth which comes about because $\partial \gamma / \partial R>0$ ( $c f$. P96, equation [3.10]).

Some general points regarding the $M$ functions are worth mentioning. As $z \rightarrow 0 M(a, b, z) \rightarrow 1$ for all $a, b$, indicating that the disk has a finite tilt at the origin. Note that since we have required $\operatorname{Re}(\sigma)>0$, the tilt is not stationary in the inertial frame $(\sigma=0$ is discussed in section 2.1). As we will see below, however, the tilt at $R=0$ for the growing modes can be extremely small. The $\operatorname{Re}(a)=1 M$ functions are the only genuinely oscillatory (i.e., zero-crossing) functions; the radial part $|M|$ is symmetric about $a=1$ for $0<\operatorname{Re}(a)<2$ but the angular dependence differs for $\operatorname{Re}(a)>1$ and $\operatorname{Re}(a)<1$. Figure 1 shows $|M| \propto|\beta|$ as a function of $|z|$ for several values of $\operatorname{Re}(a)$ with $\operatorname{Im}(a) \equiv-\omega=0$. For $\omega>0$ (corresponding to growing modes), the first zero (or minimum) of $|M|$ moves to larger $|z|$, and there is a dramatic increase in the amplitude of the first maximum of $|M|$, which also shifts to larger $|z|$. Both the magnitude of the shift and the increase in amplitude increase with $\omega$. For example, Figure 2 plots $|M|$ for $\operatorname{Re}(a)=1$ and several values of $\omega$; the behavior for other values of $\operatorname{Re}(a)$ is very similar. All the $|M|$ have been normalized to 
a maximum of one, and are labeled with $\omega$ and the normalization constant $\beta_{o}$. For $\omega=10$, the normalization constant $\beta_{o} \approx 2.5 \times 10^{-12}$.

As we show below, the maximum value $\omega_{\max }$ of $\omega$ (and therefore of the growth rate $\sigma$ ) is constrained by the outer radius of the disk (either the physical edge or the optically thick edge). There are, however, likely to be unstable modes present throughout the range $0<\omega<\omega_{\max }$. This can be seen by considering a WKB-type analysis and replacing $\partial / \partial R$ by $i k$ ( $c f$. P96). In this case we obtain

$$
\sigma=i\left\{-\Gamma_{o} k R+\frac{\frac{1}{2} \nu_{o}(k R)^{2}}{R^{1 / 2}}\right\} .
$$

Thus for a fixed $k R$ (i.e., a fixed radial mode structure) we see that $\omega \propto-\operatorname{Im}(\sigma)$ is largest, $\omega_{\max }$, at large radius. Conversely, as $R$ decreases it is evident that all unstable values of $\omega$ in the range $0<\omega<\omega_{\max }$ are present. Physically, this arises because the viscosity $\left(\nu \propto R^{3 / 2}\right)$ has a stronger radial dependence than the radiation torque parameter $(\Gamma \propto R)$. Thus for arbitrarily small $\omega$ there is always some radius at which the torque term dominates over the viscous term, and the disk is unstable to warping. In a real system, however, the development of small-scale modes will be limited by the disk age, as we discuss below.

\section{Time Evolution and Steady State}

Pringle (1996) concluded that centrally-illuminated accretion disks are unstable to warping beyond a critical radius $R_{c}$, given by

$$
\frac{R_{c}}{R_{s}}=\left(\frac{2 \sqrt{2} \pi \eta}{\epsilon}\right)^{2}
$$

the disks remain flat interior to this radius. At first glance this appears substantially different from the results of section 2, which showed that the tilt $\beta$ must be non-zero at the origin. However, the time evolution of the tilt is crucial. With increasing $\omega$, the initial growth of the warp occurs at larger radius, and the amplitude of the maximum of $\beta$ relative to the $R=0$ value grows dramatically $(c f$. Figure 2$)$; for $\operatorname{Re}(a)=1, \beta_{\max } / \beta_{o} \propto(\exp (2 \pi \omega)-1) / \omega$. Thus the disk will begin to warp initially at the largest possible radius (close to the disk physical or optical depth edge) and will be flat interior to this radius, as in Pringle's analysis. The main difference is that here the warp has non-zero precession frequency (i.e., the real part of $a$ is non-zero), whereas in P96 the growing modes were purely imaginary. Note also, from equation (6) for $z$, that if the warp begins at some value of $|z|=|z|_{w}$, this corresponds to a radius

$$
\frac{R_{w}}{R_{s}}=\frac{1}{2}\left(\frac{\eta}{\epsilon}\right)^{2}|z|_{w}^{2}
$$

which shows the same scaling with $\eta$ and $\epsilon$ as equation (4.3) of P96, given above. 
If we require that the disk be unwarped beyond some maximum radius $R_{\max }$ (as will be the case, for example, if the disk goes optically thin beyond some radius, so that the instability ceases to operate), then we must have $\operatorname{Re}(a)=1$, as this is the only mode that passes through zero. The warp then has a unique precession rate, $\operatorname{Re}(\sigma)=\Gamma_{o} / 2$. Furthermore, the requirement that the first zero of $\beta$ (and hence $|M|$ ) occur at $R_{\max }$ fixes a unique value for the the maximum value of $\omega$ (cf. Figure 2). (A similar boundary condition will determine $\omega_{\max }$ even if $a \neq 1$, since the radius of the warp onset always increases with $\omega$.)

One might expect that the steady state of such an accretion disk will be the $\omega=0$ solution. However, real accretion disks will not necessarily reach this state. The tilt $\beta$ - and therefore the amplitude of the warp - will saturate at some finite value, due to nonlinear effects which have been ignored in our analysis. This will of course occur first for the fastest growing mode, at the largest unstable radius. With time the slower-growing modes (only those with zeros at $R_{\max }$ if we require the disk to be flat there) will develop at smaller radius. However, due to the rapid reduction in amplitude $\beta_{\max } / \beta_{o}$ with decreasing $\omega$, the slower-growing modes have to evolve through a progressively larger number of $e$-folding times to reach the amplitude of the mode with $\omega=\omega_{\max }$. For $\operatorname{Re}(a)=1$, the number of required $e$-folding times is approximately $\pi\left(\omega_{\max }-\omega\right)$ (assuming $2 \pi \omega \gg 1$ ). Thus the minimum value of $\omega$ that can have developed significant amplitude is set approximately by the condition

$$
\tau_{\text {disk }}=\frac{\pi\left(\omega_{\max }-\omega_{\min }\right)}{\omega_{\min }} \tau_{i}\left(\omega_{\max }\right)
$$

where $\tau_{\text {disk }}$ is the lifetime of the accretion disk and $\tau_{i}\left(\omega_{\max }\right)$ is the timescale for growth of the mode with $\omega=\omega_{\max }$. As we will see in section 1 , this is likely to be pose a significant constraint on $\omega_{\min }$ for accretion disks around supermassive black holes in active galactic nuclei.

In addition, the effects of shadowing - which we have ignored - may be important for modes which have multiple zeros between $R=0$ and $R_{\max }$. This will depend on both the actual size of the central source of luminosity and the amplitude at which the warp eventually saturates.

Figure 3 shows the shape of the warp for the $a=1, \omega=0$ (steady-state) solution. The disk surface has been plotted out to the radius $(x=2 \pi)$ at which the warp returns to the equatorial plane. The maximum amplitude of the warp has been fixed at $10 \%$ of this radius. The spiral shape of the line of nodes is readily visible.

\section{Applications}

\subsection{The Warped Maser Disk of NGC 4258}

VLBI observations of the $22 \mathrm{GHz}$ water maser line emission from the nucleus of NGC 4258 (which possesses a low-luminosity active nucleus) have shown that the maser emission arises in a thin, warped disk, with an inner radius of $0.13 \mathrm{pc}$ and an outer radius of $0.25 \mathrm{pc}$, which exhibits 
a perfectly Keplerian velocity curve to within the measurement errors (Greenhill et al. (1995); Miyoshi et al. (1995)]). The derived central mass is $M_{c}=3.6 \times 10^{7} M_{\odot}$, and as shown by Maoz (1995), dynamical arguments essentially rule out mass models other than a supermassive black hole. The obliquity of the warp is $\mu \approx 0.25$ at the outer edge of the masing zone (Greenhill 1995, private communication) and decreases steadily inward.

Neufeld, Maloney, \& Conger (1995) showed that X-ray irradiation of dense molecular gas is an effective mechanism for generating powerful water maser emission; this also naturally explains the association of water megamasers with active galactic nuclei (Claussen \& Lo (1986); Braatz. Wilson, \& Henkel (1994)). These models also show that there is a critical pressure at which the gas goes from molecular to atomic. By modeling the NGC 4258 disk using the standard $\alpha$-prescription for viscosity, Neufeld \& Maloney (1995) (NM95) showed that the disk would be extremely thin $\left(h / R \ll 10^{-2}\right)$, that the transition from molecular to atomic gas should be identified with the outer edge of the masing region, and that the mass accretion rate through the disk is $\dot{M} / \alpha \approx 7 \times 10^{-5} M_{\odot} \mathrm{yr}^{-1}$. This mass accretion rate in turn implies that the radiative efficiency $\epsilon \approx 0.1$. The existence of the warp in this picture is crucial, since for a flat disk neither the greatly reduced irradiation from the central source nor heating by viscous dissipation would keep the disk warm enough to produce substantial amounts of water or excite the masing transitions. In fact, NM95 suggested that the inner edge of the masing disk marked the inner boundary of the warp, as is the case in the model for the warp derived by Miyoshi et al. (1995). Although an arbitrary imposed warp would be kinematically stable in a Keplerian potential, since the vertical and azimuthal frequencies are the same, the origin of the warp in NGC 4258 remained puzzling.

Here we show that the radiation-driven warping instability discovered by Pringle (1996) provides a plausible explanation for the warping of the disk. From equation (6) for $z$, with $R_{s} \simeq 1.1 \times 10^{13} \mathrm{~cm}$ for the central object in NGC 4258, we find that the inner and outer edges of the masing region are at

$$
|z|_{\text {in }} \approx 28 \epsilon_{0.1}, \quad|z|_{\text {out }} \approx 39 \epsilon_{0.1}
$$

where $\epsilon=0.1 \epsilon_{0.1}$. As can be seen from Figure 2, this requires $\omega_{\max } \sim 10$ for $\epsilon_{0.1} \sim 1$, as is indicated by the results of NM95. (We note that in the molecular zone at the outer edge of the masing region in the model of NM95, the hydrogen column density through the disk $N_{H} \sim 10^{24} \mathrm{~cm}^{-2}$, satisfying the assumption that the disk is optically thick to both absorption and emission. Also, the outer edge of the masing zone does not have to coincide with the maximum of the warp.) It is likely that $\omega_{\min }$ is not much smaller than this value, due to the limit set by the finite age of the accretion disk. The characteristic timescale for growth of the instability is

$$
\tau_{i}(\omega) \approx \frac{1}{|\sigma|}=\frac{2}{\omega \Gamma_{o}}
$$

assuming that $\omega \gg \operatorname{Re}(a)$. This can be expressed in terms of the viscous timescale $\tau_{\text {visc }} \sim 2 \pi R^{2} \Sigma / \dot{M}:$

$$
\tau_{i}(\omega) \sim \frac{12}{\omega}|z|^{-1} \eta^{-1} \tau_{\text {visc }}
$$


which, under the assumption that the luminosity is accretion-powered, depends only on $\epsilon$ and not on $L$ and $\dot{M}$ individually. Assuming standard $\alpha$-viscosity and negligible self-gravity for the accretion disk, this becomes

$$
\tau_{i}(\omega) \sim \frac{4}{\omega} \frac{c R_{s}}{\alpha c_{s}^{2} \epsilon} \approx 3 \times 10^{7} \frac{M_{8}}{\omega \alpha T_{3} \epsilon_{0.1}} \mathrm{yrs}
$$

where $M_{c}=10^{8} M_{8} M_{\odot}, c_{s}$ is the sound speed, the gas temperature $T=10^{3} T_{3} \mathrm{~K}$, and the numerical coefficient is for molecular gas. In the masing molecular zone, $0.3<T_{3}<1$. While the characteristic growth time for the $\omega=10$ mode in NGC 4258 is $\tau_{i} \sim 5 \times 10^{6}$ years, the time required for even the $\omega=5$ mode to become comparable in amplitude is an order of magnitude longer. Thus we expect that the warp will be dominated by modes with $\omega$ close to $\omega_{\max }$, unless the accretion disk is extremely long-lived $\left(\tau_{\text {disk }} \gg 10^{8}\right.$ years $)$. Hence the predictions of the radiation-driven warp instability model are in reasonable agreement with the observations of NGC 4258 , for $\epsilon \sim 0.1$.

This instability cannot provide an explanation for the warp if NGC 4258 possesses an advection-dominated accretion disk with $\epsilon \sim 10^{-3}$, as suggested by Lasota et al. (1996). From equation (17), this requires that $|z|_{\text {in }},|z|_{\text {out }} \ll 1$. However, the only modes that have any power at such small $|z|$ have $\omega<1$. Equation (20) shows that the timescale for growth of these modes is prohibitively long, with $\tau_{i}>10^{9}$ years.

\subsection{The 164-day Precession Period of SS 433}

SS 433 is an eclipsing binary system containing an early-type star and an accretion disk around a compact object. The optical and infrared spectrum shows two sets of highly Doppler-shifted emission lines, which reach velocity extremes of $+50,000$ and $-30,000 \mathrm{~km} \mathrm{~s}^{-1}$, respectively. These emission lines are believed to arise from oppositely-directed relativistic $(v / c \approx 0.26)$ beams of material emanating from SS 433 (reviews of observations and models of SS 433 can be found in Margon (1984) and Zwitter et al. (1989)]). The high-velocity systems show systematic changes in wavelength, with a period of approximately 164 days. This period is generally regarded to be the result of precession of the ejection axis of the system. There is also direct evidence for precession of the beams from radio maps (Hjellming \& Johnston (1986)] . In addition, there is evidence for precession of the accretion disk, which dominates the optical light of the system, from optical photometry (Margon (1984).

A number of models have been proposed to explain the precession of the accretion disk. Most of these involve "slaved" precession, in which the residence time in the disk is sufficiently short that the inner disk follows the precessional motion of a misaligned companion star or outer disk (e.g., Katz et al. 1982). This in turn requires that the disk be very thick, if the internal disk pressure is to be comparable to the very large viscous stresses which are required. Support for the existence of a thick $(h / R \sim 1)$ disk is provided by analysis of the light curve (Anderson, Margon, 
\& Grandi (1983)).

However, if the accretion disk in SS 433 is substantially warped, the actual thickness of the disk may be much smaller than inferred from the light curve, which will measure the amplitude of the warp. (This is certainly the case in NGC 4258, in which the solid angle occupied by the disk about the source is completely dominated by the warp.) As shown in section 2, the real part of $a$ is in general not zero, so the warp will precess with a fixed pattern speed. Assuming that the accretion disk in SS 433 is subject to the radiation-driven warp instability, we now show that that the 164-day precession period can be explained as the result of precession of the warp.

We again assume that $\operatorname{Re}(a)=1$, so that we can require the outer boundary of the disk to be unwarped. As noted earlier, this fixes the precession rate at $\Gamma_{o} / 2$ and so the precession timescale is just $T_{\text {prec }} \approx 4 \pi / \Gamma_{o}$. Since the assumption of an isothermal disk is likely to be poor for SS 433 , we instead write the viscosity in the form

$$
\nu_{1}=\frac{\alpha}{\sqrt{2}} c R\left(\frac{R}{R_{s}}\right)^{-1 / 2}\left(\frac{h}{R}\right)^{2}
$$

and so the precession period is

$$
T_{\text {prec }} \approx \frac{16 \pi}{\alpha \epsilon} \frac{R}{c}\left(\frac{R}{h}\right)^{2}
$$

where this should be evaluated at $R=R_{\text {warp }}$. For accretion disks around $M_{c} \sim$ solar-mass objects, the timescales for growth of the unstable modes are sufficiently short that the warp reaches the steady-state, $\omega=0$ solution. For $a=1$ the first maximum occurs at $|z| \approx 1.3 \pi$, and we take this to correspond to $R_{\mathrm{warp}}$. Thus the precession timescale becomes

$$
T_{\text {prec }} \sim 430 \frac{\eta^{2}}{\alpha \epsilon^{3}} \frac{R_{s}}{c}\left(\frac{R}{h}\right)_{\mathrm{R}_{\mathrm{warp}}}^{2} .
$$

For SS 433, $\epsilon$ is probably $\ll 0.1$, since the accretion rate is almost certainly substantially super-Eddington (e.g., Begelman et al. (1980)] ) and so the precession timescale is

$$
T_{\text {prec }} \sim 50 \frac{\eta^{2}}{\alpha \epsilon_{0.001}^{3}}\left(\frac{M_{c}}{M_{\odot}}\right)\left(\frac{R}{h}\right)_{\mathrm{R}_{\text {warp }}}^{2} \text { days }
$$

which can explain the 164-day precession period in SS 433 for reasonable values of $\alpha$ and $(R / h)$. We also note that the precession can be either prograde or retrograde with respect to the direction of disk rotation, as can be seen from the derivation leading to equation (2.18) of P96.

\section{Summary}

Our global analysis confirms the conclusion of Pringle (1996) that thin, centrally-irradiated accretion disks are generically unstable to warping driven by radiation pressure. The maximum growth rate is set by the largest unstable radius (either the physical boundary of the disk or the 
radius at which it becomes optically thin to the bulk of the incident or re-emitted flux). The growth rate on smaller scales in accretion disks around supermassive black holes in active galactic nuclei will in general be limited by the lifetime of the accretion disk. Although the final state of the instability will be determined by nonlinear processes which have not been considered in this analysis, we have shown that the warping instability provides an explanation for the existence of the warp in the masing accretion disk in NGC 4258. Furthermore, for plausible parameters the 164-day precession period in SS 433 can be identified with the precession period of the warp, as can the long (30 - 300 day) periods seen in other X-ray binary systems, such as Her X-1 (see White, Nagase, \& Parmar 1995 for a review).

Radiation-driven warping and precession thus offers a robust mechanism for producing warped, precessing accretion disks in AGN and in accreting binary systems such as Her X-1 and SS 433. Because the warp adjusts itself in such a way as to precess at a uniform rate at all radii, radiation-driven warping is an inherently global mechanism, thereby avoiding the difficulties inherent in other proposed mechanisms for producing warping and precession. This instability should generally be important in thin accretion disks.

PRM acknowledges support through the NASA Long Term Astrophysics Program under grant NAGW-4454. MCB was supported by NSF grant AST 91-20599.

\section{REFERENCES}

Abramowitz, M.A., \& Stegun, I. 1964, Handbook of Mathematical Functions, AMS 52 (Washington: U.S. Government Printing Office)

Anderson, S.F., Margon, B., \& Grandi, S.A. 1983, ApJ, 269, 605

Begelman, M.C., Sarazin, C.L., Hatchett, S.P., McKee, C.F., \& Arons, J. 1980, ApJ, 238, 722

Braatz, J.A., Wilson, A.S., \& Henkel, C. 1994, ApJ, 437, L99

Claussen, M.J., \& Lo, K.-Y. 1986, ApJ, 308, 592

Greenhill L. J., Jiang, D. R., Moran, J. M., Reid, M. J., Lo, K. Y., \& Claussen M. J. 1995b, ApJ, 440,619

Hjellming, R.M., \& Johnston, K.J. 1986, in The Physics of Accretion onto Compact Objects, ed. K.O. Mason, M.G. Watson, N.E. White (Berlin: Springer-Verlag), p. 287

Katz, J.I., Anderson, S.F., Margon, B., \& Grandi, S. A. 1982, ApJ, 260, 780

Lasota, J.P., Abramowicz, M.A., Chen, X., Krolik, J., Narayan, R., \& Yi, I. 1996, ApJ, in press.

Maoz, E. 1995, ApJ, 447, L91 
Margon, B. 1984, ARA\&A, 22, 507

Miyoshi, M., Moran, J., Herrnstein, J., Greenhill, L., Nakai, N., Diamond, P., \& Inoue, M. 1995, Nature 373, 127

Neufeld, D.A., Maloney, P.R., \& Conger, S. 1994, ApJ, 436, L127

Neufeld, D.A., \& Maloney, P.R. 1995, ApJ, 447, L19

Petterson, J.A. 1977, ApJ, 216, 827

Pringle, J.E. 1992, MNRAS, 258, 811

Pringle, J.E. 1996, MNRAS, in press.

White, N.E., Nagase, F., \& Parmar, A.N. 1995, in X-Ray Binaries, ed. W.H.G. Lewin, J. van Paradijs, \& E.P.J. van den Heuvel (Cambridge: Cambridge University Press), p. 1

Zwitter, T., Calvani, M., Bodo, G., \& Massaglia, S. 1989, Fund. Cosmic Phys. 13, 309 
Fig. 1.- The absolute magnitude of the Kummer function $M(a, 2, z)$ for $\omega=-\operatorname{Im}(a)=0$ and several values of $\operatorname{Re}(a)$ (i.e., the time-independent solutions of the twist equation). The magnitude of the local angle of tilt $|\beta|$ is equal to $|M|$ times a normalization coefficient. The radial variable $z$ is defined by $z=i \sqrt{2} \epsilon\left(R / R_{s}\right)^{1 / 2} / \eta$, where $\epsilon$ is the radiative efficiency $\epsilon=L / \dot{M} c^{2}, R_{s}$ is the Schwarzschild radius, and $\eta$ is the ratio of vertical to radial viscosities. The Kummer function with $\operatorname{Re}(a)=1$ is the only zero-crossing function. For other values of $a|M|$ is symmetric about $a=1$. Requiring that the tilt $\beta \rightarrow 0$ as $R \rightarrow \infty$ requires that the real part of $a$ satisfy $0<\operatorname{Re}(a)<2$.

Fig. 2. - The amplitude of the local angle of tilt $|\beta|$ for several of the growing solutions of the twist equation. The Kummer functions $|M(1-i \omega, 2, z)|$ are plotted. All these modes have $\operatorname{Re}(a)=1$ and grow as $e^{\omega t}$. They have all been normalized so that the maximum amplitude is 1 ; each curve is labeled with $\omega$ and with the normalization constant $\beta_{o}$, i.e., the magnitude of the tilt at the origin. For $\omega \gg 1$ this is extremely small, so that for modes with high growth rates the disks remain flat interior to a critical radius. The maximum value of $\omega$ for a disk is fixed by the largest unstable radius, which is either the physical edge of the disk or the radius at which it becomes optically thin.

Fig. 3. - Surface plot of the warp shape for the steady-state $(\omega=0), a=1$ mode of an isothermal accretion disk. The warp has been plotted out to the radius at which it returns to the equatorial plane; the amplitude of the warp has been fixed at $10 \%$ of this radius. 


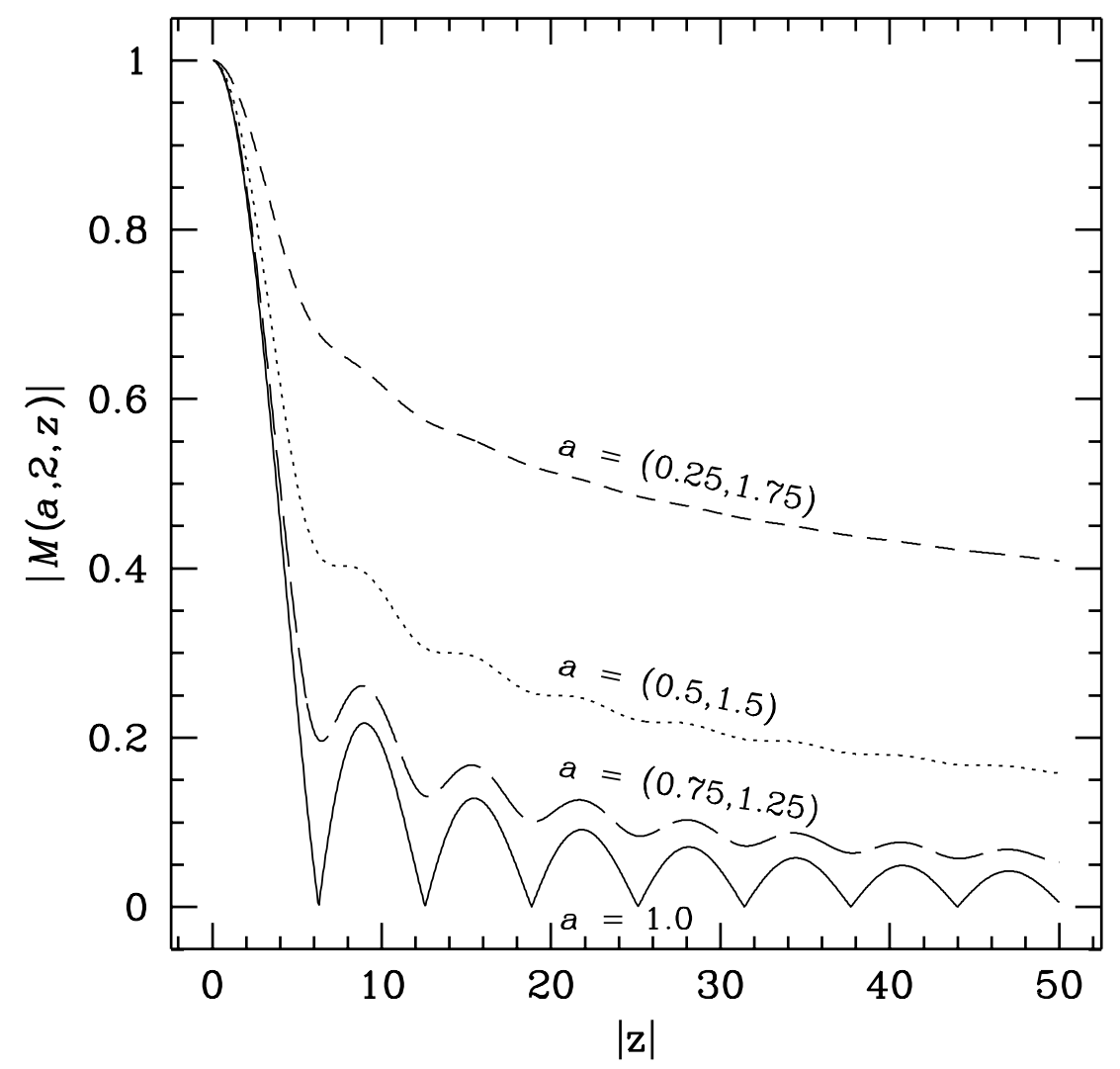




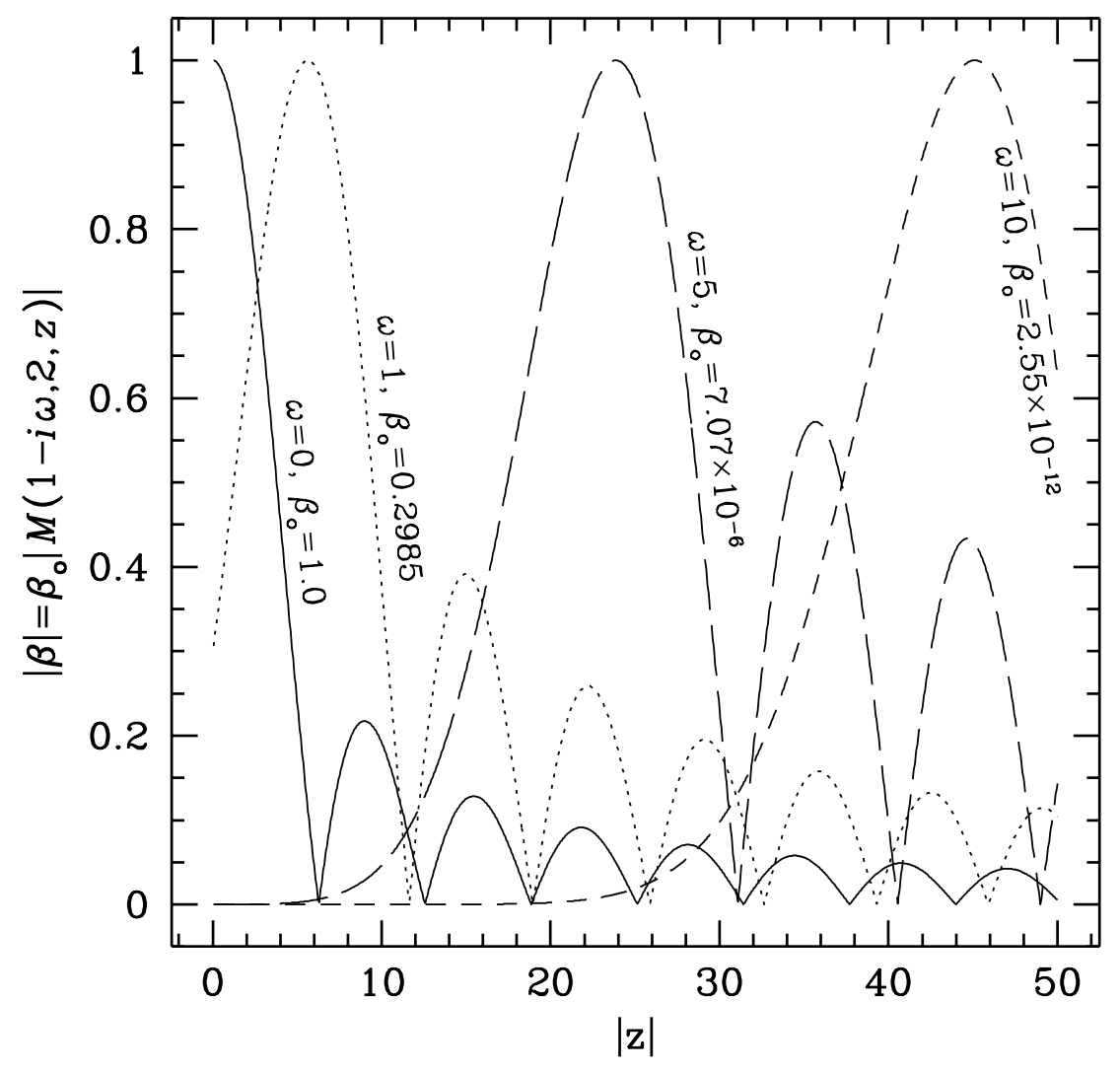




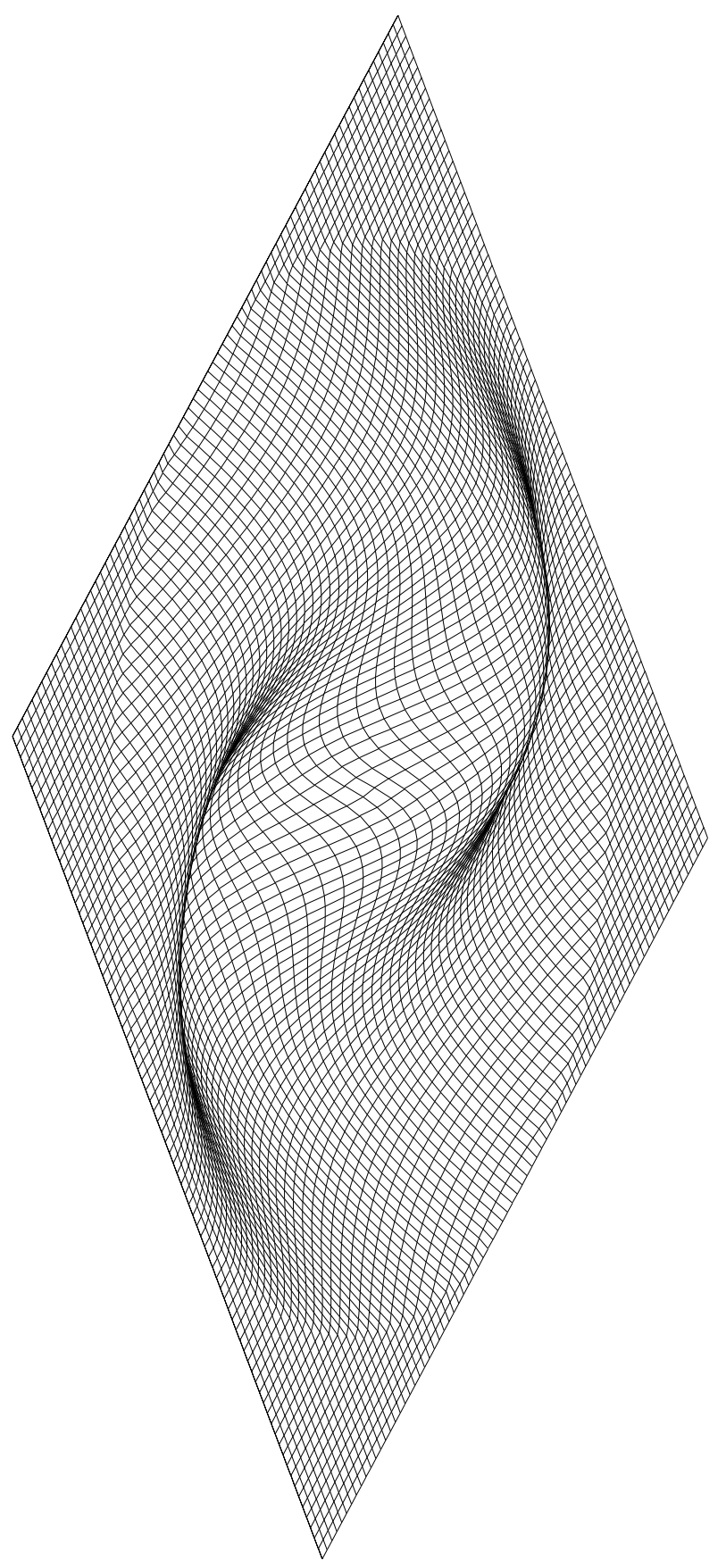

